\title{
Patterns of antihypertensive Drug Utilization among the Cardiologists of Bangladesh in Initiating Hypertension Treatment
}

\author{
AAS Majumder \\ Department of Cardiology, National Institute of Cardiovascular Diseases, Dhaka
}

\begin{abstract}
:
Keywords:

Hypertension,

Background: Cardiologists play the major role in the management of hypertension in Bangladesh.

Anti Different classes of antihypertensive drugs are being used by the cardiologists in initiating hypertension treatment depending on the age and gender of the patient, severity of hypertension and associated diseases. However, there is no particular study to evaluate the patterns of the utilization

hypertensive drugs of these antihypertensive drugs. Again, it is very important to use the right antihypertensive drug for the right patient to move further ahead in the management of hypertension. Hence, the current use of antihypertensive drugs in initiating hypertension treatment was analyzed.
\end{abstract}

Methods: This was a nationwide survey with a participation of 196 cardiologists. Individual opinions from these cardiologists were collected through a questionnaire asking which antihypertensive they use most in initiating hypertension treatment. Collected data were analyzed by SPSS 17 software.

Results: ACEI mono-therapy is the mostly used antihypertensive drug class for male patients aged $<55$ years having mild to moderate hypertension. CCB mono-therapy is the mostly used antihypertensive drug class for female patients aged < 55 years having mild to moderate hypertension. CCB mono-therapy is also the mostly used antihypertensive drug class for both male and female patients aged $e^{\prime} 55$ years having mild to moderate hypertension. For severe hypertensive patients aged < 55 years, the mostly used antihypertensive drugs are combinations of $A C E I+C C B+D$ for both male and females. For severe hypertensive patients aged e" 55 years, the mostly used antihypertensive drugs are combinations of $A C E I+C C B+D$ for males and combinations of $C C B+B B$ for females. However, the overall pattern of using antihypertensive drugs did not significantly vary between male and female patients.

Conclusion: The pattern of using antihypertensive drugs varies depending on age and gender of the patient and on the severity of hypertension. But, the overall pattern of using antihypertensive drugs did not significantly vary between male and female patients.

(Cardiovasc.j. 2012; 4(2): 114-119)

\section{Introduction:}

Hypertension affects approximately one billion individuals worldwide and is a major risk factor for coronary heart disease, heart failure, cerebrovascular disease and chronic renal failure. ${ }^{1,2}$ It has identified as the leading global risk factor for mortality and as the third leading risk factor for disease burden. ${ }^{3}$ Hence, Hypertension is an important public health challenge worldwide.

The prevalence of hypertension is expected to be high in Bangladesh as well. However, there is few data available in this regard. The latest one is a clinic-based survey done in 2004 by Zaman et. al. In this survey, around $17.8 \%$ of the total patient population aged $>20$ years was found hypertensive ${ }^{4}$.

Globally, cardiologists play the central role in managing hypertensive patients ${ }^{5}$. The situation is same in Bangladesh. The primary goal of the hypertension management is to achieve the maximum reduction in the long-term total risk of cardiovascular morbidity and mortality. ${ }^{6}$ To achieve this goal, commonly 5 classes of antihypertensive drugs, namely â-blockers (BB), Diuretics (D), Calcium Channel Blockers (CCB),

Address for Correspondence: Prof. Abdullah Al Shafi Majumder, Department of Cardiology, National Institute of Cardiovascular Diseases, Dhaka, Bangladesh. E-mail: aasm@bangla.net. 
Angiotensin Converting Enzyme Inhibitors (ACEI) and Angiotensin Receptor Blockers (ARB), are being used by the cardiologists in initiating hypertension treatment depending on the age and gender of the patient, severity of hypertension and associated diseases. While choosing a specific antihypertensive drug or a drug combination one should consider the factors like the previous favorable or unfavorable experience of the individual patient with a given class of drugs, the effect of drugs on cardiovascular risk factors in relation to the cardiovascular risk profile of the individual patient, the presence of sub-clinical organ damage, clinical cardiovascular disease, renal disease or diabetes which may be more favorably treated by some drugs than others, the presence of other disorders that may limit the use of particular classes of antihypertensive drugs, the possibilities of interactions with drugs used for other conditions and the cost of drugs. ${ }^{6}$ Again, it is very important to use the right antihypertensive drug for the right patient to achieve the goals of hypertension management. Therefore, the current study was carried out with an objective to evaluate the patterns of the utilization of the commonly used antihypertensive drugs.

\section{Methods}

\section{Procedure:}

During the summer 2010, a questionnaire with a cover letter was sent to 441 cardiologists working in different cities of Bangladesh. They were requested to provide their opinions on which antihypertensive they use most in initiating hypertension treatment depending on age and gender of the patient and on the severity of hypertension (mild, moderate and severe). Mild, moderate and severe hypertension was defined as the patients having a blood pressure range of 140/ 90 to $159 / 99,160 / 100$ to $179 / 109$ and $\geq 180 / 110$

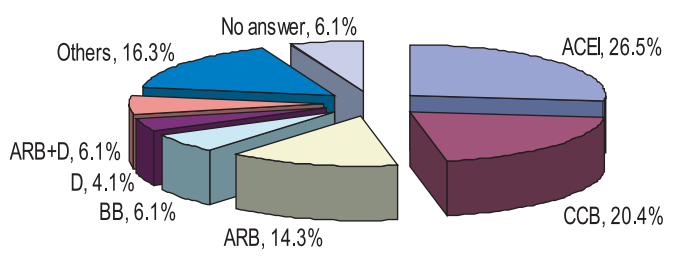

Fig.1(A): Antihypertensive utilization in $<55$ years old mild hypertensive patients (Male)
$\mathrm{mmHg}$ respectively. A total 200 cardiologists gave their opinion. Among the 200 filled-in questionnaires, 4 were excluded due to ambiguity in information and data of 196 filled-in questionnaires were analyzed.

Statistical analysis:

Data were analyzed by SPSS 17 software. Antihypertensive drugs usage between male and female in mild, moderate and severe hypertension was compared and the level of significance was calculated by f-test. All statistical tests were onetailed and all analyses were considered statistically significant if $\mathrm{p}<0.05$.

\section{Results:}

Antihypertensive utilization in the patients $<55$ years and patients $\geq 55$ years are summarized in Table \# 1 and Table \# 2 respectively.

\section{Patients $<55$ years}

In case of $<55$ years old mild hypertensive patients, the mostly used antihypertensive drugs are ACEI (26.5\%) for males and CCB (24.5\%) for females (Figure \# 1A and 1B). However, the overall pattern of using antihypertensive drugs did not significantly vary between male and female patients $(p=0.93)$. $71.4 \%$ of respondent uses mono-therapy while $22.4 \%$ uses combinations and rest $6.1 \%$ did not answer this question. The percentages of using mono-therapy or combination and of no answer are same for both male and female patients.

In case of $<55$ years old moderate hypertensive patients, the mostly used antihypertensive drugs are ACEI (18.9\%) for males and CCB (20.4\%) for females (Figure\# 2A and 2B). However, the overall pattern of using antihypertensive drugs did not significantly vary between male and female patients $(p=0.90)$. For male patients, $60.2 \%$ of respondent uses mono-therapy while $34.7 \%$ uses

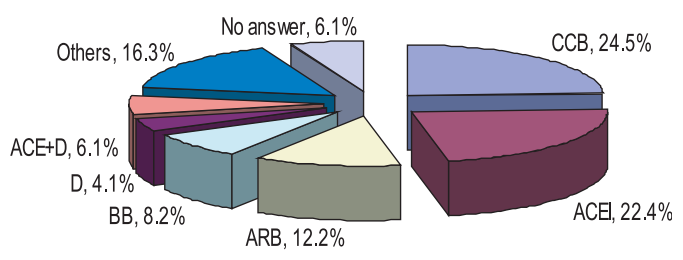

Fig.1(B): Antihypertensive utilization in < 55 years old mild hypertensive patients (Female) 


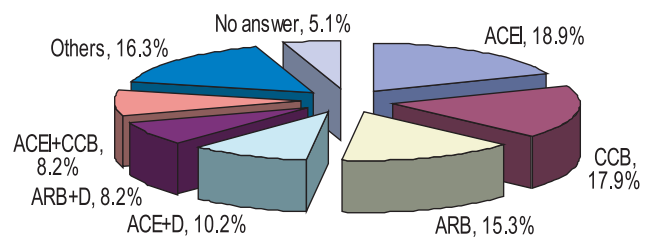

Fig.-2 (A): Antihypertensive utilization in $<55$ years old moderate hypertensive patients (Male)

combinations and rest $5.1 \%$ did not answer this question. For female patients, $63.3 \%$ of respondent uses mono-therapy while $34.7 \%$ uses combinations and rest $2.0 \%$ did not answer this question.

In case of $<55$ years old severe hypertensive patients, the mostly used antihypertensive drugs are combinations of $\mathrm{ACEI}+\mathrm{CCB}+\mathrm{D}$ for both male

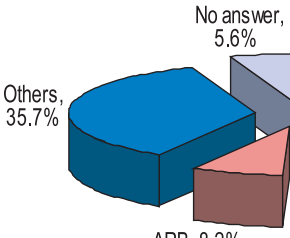

ARB, $8.2 \%$

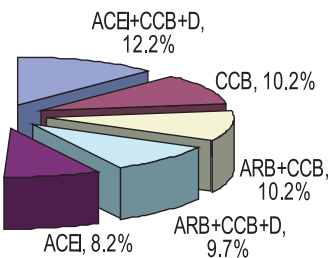

Fig.-3 (A): Antihypertensive utilization in $<55$ years old severe hypertensive patients (Male).

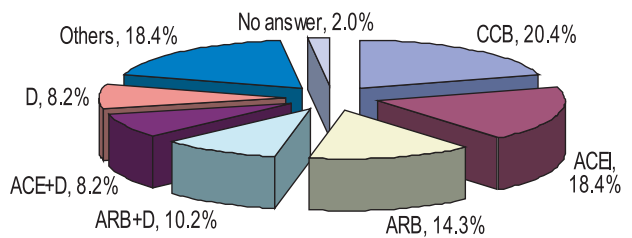

Fig.-2 (B): Antihypertensive utilization in < 55 years old moderate hypertensive patients (Female)

and female patients (Figure \# 3A and 3B). The overall pattern of using antihypertensive drugs did not significantly vary between male and female patients $(p=0.56)$. For male patients, $30.6 \%$ of respondent uses mono-therapy while $63.8 \%$ uses combinations and rest $5.6 \%$ did not answer this question. For female patients, $36.7 \%$ of respondent

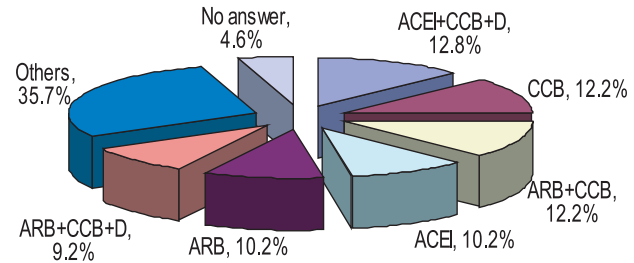

Fig.-3 (B): Antihypertensive utilization in $<55$ years old severe hypertensive patients (Female)

Table-I

Utilization of antihypertensive drugs in initiating hypertension treatment for the patient $<55$ years

\begin{tabular}{|c|c|c|c|c|c|c|c|c|c|c|}
\hline & & \multicolumn{2}{|c|}{ Mild Hypertension } & \multirow[b]{2}{*}{$\mathrm{p}$ value* } & \multicolumn{2}{|c|}{ Moderate Hypertension } & \multicolumn{4}{|c|}{ Severe Hypertension } \\
\hline & & Male & Female & & Male & Female & $\mathrm{p}$ value* & Male & Female & $p$ value* \\
\hline \multirow[t]{5}{*}{ Mono-therapy } & ACEI & $52(26.5 \%)$ & $44(22.4 \%)$ & 0.93 & $37(18.9 \%)$ & $36(18.4 \%)$ & 0.90 & $16(8.2 \%)$ & $20(10.2 \%)$ & 0.56 \\
\hline & $\mathrm{ARB}$ & $28(14.3 \%)$ & $24(12.2 \%)$ & & $30(15.3 \%)$ & $28(14.3 \%)$ & & $16(8.2 \%)$ & $20(10.2 \%)$ & \\
\hline & $\mathrm{CCB}$ & $40(20.4 \%)$ & $48(24.5 \%)$ & & $35(17.9 \%)$ & $40(20.4 \%)$ & & $20(10.2 \%)$ & $24(12.2 \%)$ & \\
\hline & BB & $12(6.1 \%)$ & $16(8.2 \%)$ & & $4(2.0 \%)$ & $4(2.0 \%)$ & & - & - & \\
\hline & $\mathrm{D}$ & $8(4.1 \%)$ & $8(4.1 \%)$ & & $12(6.1 \%)$ & $16(8.2 \%)$ & & $8(4.1 \%)$ & $8(4.1 \%)$ & \\
\hline \multirow[t]{9}{*}{ Combination } & $\mathrm{ACE}+\mathrm{D}$ & $8(4.1 \%)$ & $12(6.1 \%)$ & & $20(10.2 \%)$ & $16(8.2 \%)$ & & $16(8.2 \%)$ & $16(8.2 \%)$ & \\
\hline & $\mathrm{ACEI}+\mathrm{CCB}$ & - & - & & $16(8.2 \%)$ & $12(6.1 \%)$ & & $16(8.2 \%)$ & $8(4.1 \%)$ & \\
\hline & $\mathrm{ACEI}+\mathrm{ARB}$ & $12(6.1 \%)$ & $12(6.1 \%)$ & & $4(2.0 \%)$ & $4(2.0 \%)$ & & - & - & \\
\hline & $\mathrm{ARB}+\mathrm{D}$ & $12(6.1 \%)$ & $8(4.1 \%)$ & & $16(8.2 \%)$ & $20(10.2 \%)$ & & $14(7.1 \%)$ & $12(6.1 \%)$ & \\
\hline & $\mathrm{ARB}+\mathrm{CCB}$ & $8(4.1 \%)$ & $8(4.1 \%)$ & & $4(2.0 \%)$ & $8(4.1 \%)$ & & $20(10.2 \%)$ & $24(12.2 \%)$ & \\
\hline & $\mathrm{CCB}+\mathrm{BB}$ & $4(2.0 \%)$ & $4(2.0 \%)$ & & $8(4.1 \%)$ & $8(4.1 \%)$ & & $16(8.2 \%)$ & $12(6.1 \%)$ & \\
\hline & $\mathrm{ACEI}+\mathrm{CCB}+\mathrm{I}$ & $\mathrm{D}$ & - & & - & - & & $24(12.2 \%)$ & $25(12.8 \%)$ & \\
\hline & $\mathrm{ARB}+\mathrm{CCB}+\mathrm{D}$ & - & - & & - & - & & 19 (9.7\%) & $18(9.2 \%)$ & \\
\hline & No answer & $12(6.1 \%)$ & $12(6.1 \%)$ & & $10(5.1 \%)$ & $4(2.0 \%)$ & & $11(5.6 \%)$ & $9(4.6 \%)$ & \\
\hline
\end{tabular}

$\mathrm{ACEI}=$ Angiotensin Converting Enzyme Inhibitor, ARB = Angiotensin Receptor Blocker, CCB = Calcium Channel Blocker, BB = Beta Blocker, $\mathrm{D}=$ Diuretic

*Level of significance comparing antihypertensive drugs usage between male and female 
Patterns of antihypertensive Drug Utilization among the Cardiologists

AAS Majumder

Table-II

Utilization of antihypertensive drugs in initiating hypertension treatment for the patient $\geq 55$ years

\begin{tabular}{|c|c|c|c|c|c|c|c|c|c|c|}
\hline & & \multicolumn{2}{|c|}{ Mild Hypertension } & \multirow[b]{2}{*}{$p$ value* } & \multicolumn{2}{|c|}{ Moderate Hypertension } & \multicolumn{4}{|c|}{ Severe Hypertension } \\
\hline & & Male & Female & & Male & Female & $\mathrm{p}$ value* & Male & Female & $\mathrm{p}$ value* \\
\hline \multirow[t]{5}{*}{ Mono-therapy } & ACEI & $28(14.3 \%)$ & $32(16.3 \%)$ & 0.55 & $32(16.3 \%)$ & $29(14.8 \%)$ & 0.74 & $8(4.1 \%)$ & $8(4.1 \%)$ & 0.51 \\
\hline & $\mathrm{ARB}$ & $24(12.2 \%)$ & $32(16.3 \%)$ & & $20(10.2 \%)$ & $12(6.1 \%)$ & & $20(10.2 \%)$ & $24(12.2 \%)$ & \\
\hline & $\mathrm{CCB}$ & $44(22.4 \%)$ & $48(24.5 \%)$ & & $36(18.4 \%)$ & $35(17.9 \%)$ & & $12(6.1 \%)$ & $16(8.2 \%)$ & \\
\hline & BB & $16(8.2 \%)$ & $12(6.1 \%)$ & & - & - & & $4(2.0 \%)$ & - & \\
\hline & $\mathrm{D}$ & $28(14.3 \%)$ & $32(16.3 \%)$ & & $16(8.2 \%)$ & $12(6.1 \%)$ & & $16(8.2 \%)$ & $12(6.1 \%)$ & \\
\hline \multirow[t]{9}{*}{ Combination } & $\mathrm{ACE}+\mathrm{D}$ & $8(4.1 \%)$ & $4(2.0 \%)$ & & $20(10.2 \%)$ & $20(10.2 \%)$ & & $20(10.2 \%)$ & $20(10.2 \%)$ & \\
\hline & $\mathrm{ACEI}+\mathrm{CCB}$ & $4(2.0 \%)$ & $4(2.0 \%)$ & & $12(6.1 \%)$ & $16(8.2 \%)$ & & $12(6.1 \%)$ & - & \\
\hline & $\mathrm{ACEI}+\mathrm{ARB}$ & $4(2.0 \%)$ & $4(2.0 \%)$ & & $12(6.1 \%)$ & $12(6.1 \%)$ & & - & - & \\
\hline & $\mathrm{ARB}+\mathrm{D}$ & - & - & & $8(4.1 \%)$ & $8(4.1 \%)$ & & $20(10.2 \%)$ & $16(8.2 \%)$ & \\
\hline & $\mathrm{ARB}+\mathrm{CCB}$ & $8(4.1 \%)$ & - & & $8(4.1 \%)$ & $16(8.2 \%)$ & & $4(2.0 \%)$ & $8(4.1 \%)$ & \\
\hline & $\mathrm{CCB}+\mathrm{BB}$ & $12(6.1 \%)$ & $12(6.1 \%)$ & & $12(6.1 \%)$ & $8(4.1 \%)$ & & $16(8.2 \%)$ & $30(15.3 \%)$ & \\
\hline & $\mathrm{ACEI}+\mathrm{CCB}+$ & $+\mathrm{D}$ & - & & $4(2.0 \%)$ & $4(2.0 \%)$ & & $28(14.3 \%)$ & $20(10.2 \%)$ & \\
\hline & $\mathrm{ARB}+\mathrm{CCB}+\mathrm{I}$ & $\mathrm{D}$ & - & & $4(2.0 \%)$ & $8(4.1 \%)$ & & $24(12.2 \%)$ & $26(13.3 \%)$ & \\
\hline & No answer & $20(10.2 \%)$ & $16(8.2 \%)$ & & $12(6.1 \%)$ & $16(8.2 \%)$ & & $12(6.1 \%)$ & $16(8.2 \%)$ & \\
\hline
\end{tabular}

$\mathrm{ACEI}=$ Angiotensin Converting Enzyme Inhibitor, $\mathrm{ARB}=$ Angiotensin Receptor Blocker, $\mathrm{CCB}=$ Calcium Channel Blocker, $\mathrm{BB}=\mathrm{Beta} \mathrm{Blocker}$, $\mathrm{D}=$ Diuretic

*Level of significance comparing antihypertensive drugs usage between male and female

uses mono-therapy while $58.7 \%$ uses combinations and rest $4.6 \%$ did not answer this question.

Patients $\geq 55$ years

In case of $\geq 55$ years old mild hypertensive patients, the mostly used antihypertensive drugs are CCB for both male and female patients (Figure \# 4(A) and 4(B). The overall pattern of using antihypertensive drugs did not significantly vary between male and female patients $(p=0.55)$. For male patients, $71.4 \%$ of respondent uses mono-therapy while $18.4 \%$ uses combinations and rest $10.2 \%$ did not answer this question. For female patients, $79.6 \%$ of respondent uses mono-therapy while $12.2 \%$ uses combinations and rest $8.2 \%$ did not answer this question.

In case of $\geq 55$ years old moderate hypertensive patients, the mostly used antihypertensive drugs

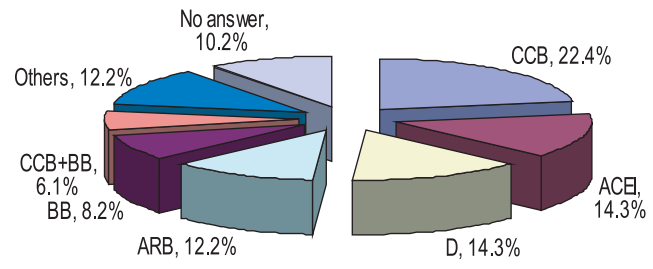

Fig.-4 (A): Antihypertensive utilization in $>55$ years old mild hypertensive patients (Male) are CCB for both male and female patients (Figure \# 5(A) and 5(A)). The overall pattern of using antihypertensive drugs did not significantly vary between male and female patients $(p=0.74)$. For male patients, $53.1 \%$ of respondent uses monotherapy while $40.8 \%$ uses combinations and rest $6.1 \%$ did not answer this question. For female patients, $44.9 \%$ of respondent uses mono-therapy while $46.9 \%$ uses combinations and rest $8.2 \%$ did not answer this question.

In case of $\geq 55$ years old severe hypertensive patients, the mostly used antihypertensive drugs are combinations of $\mathrm{ACEI}+\mathrm{CCB}+\mathrm{D}$ for males $(14.3 \%)$ and combinations of $\mathrm{CCB}+\mathrm{BB}$ (15.3\%) for females (Figure\#6(A) and 6(B). However, the overall pattern of using antihypertensive drugs did not significantly vary between male and female patients $(p=0.51)$.

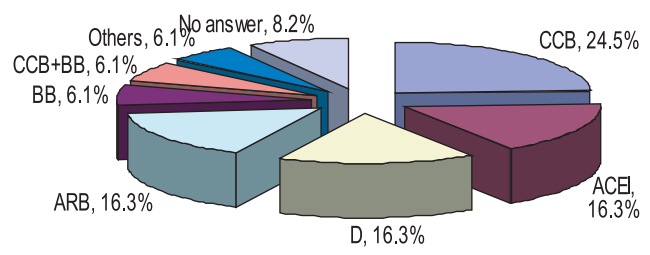

Fig.-4 (B): Antihypertensive utilization in $>55$ years old mild hypertensive patients (Female) 


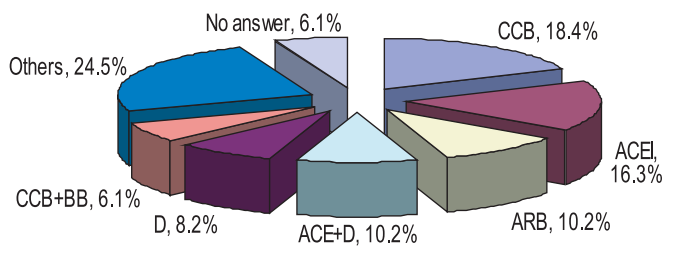

Fig.-5 (A): Antihypertensive utilization in $>55$ years old moderate hypertensive patients (Male)

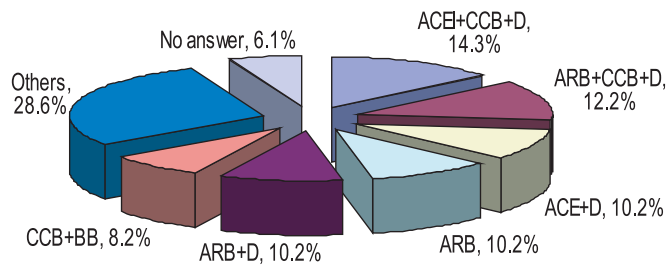

Fig.-6 (A): Antihypertensive utilization in $>55$ years old severe hypertensive patients (Male)

For male patients, $30.6 \%$ of respondent uses monotherapy while $63.3 \%$ uses combinations and rest $6.1 \%$ did not answer this question. For female patients, $30.6 \%$ of respondent uses mono-therapy while $61.2 \%$ uses combinations and rest $8.2 \%$ did not answer this question.

\section{Discussion:}

In this survey we have found that ACEI monotherapy is the mostly used antihypertensive drug class for male patients aged $<55$ years having mild to moderate hypertension. CCB mono-therapy is the mostly used antihypertensive drug class for female patients aged $<55$ years having mild to moderate hypertension. CCB mono-therapy is also the mostly used antihypertensive drug class for both male and female patients aged $\geq 55$ years having mild to moderate hypertension. For severe hypertensive patients aged $<55$ years, the mostly used antihypertensive drugs are combinations of $\mathrm{ACEI}+\mathrm{CCB}+\mathrm{D}$ for both male and females. For severe hypertensive patients aged $\geq 55$ years, the mostly used antihypertensive drugs are combinations of $\mathrm{ACEI}+\mathrm{CCB}+\mathrm{D}$ for males and combinations of $\mathrm{CCB}+\mathrm{BB}$ for females. However, the overall pattern of using antihypertensive drugs did not significantly vary between male and female patients.

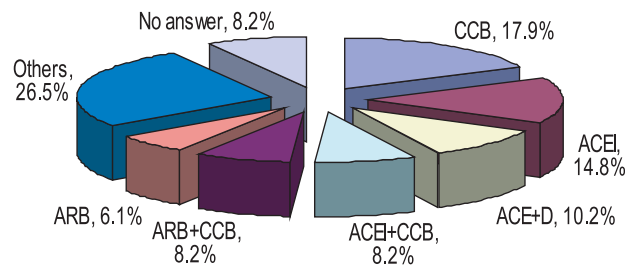

Fig.-5 (B): Antihypertensive utilization in $>55$ years old moderate hypertensive patients (Female)

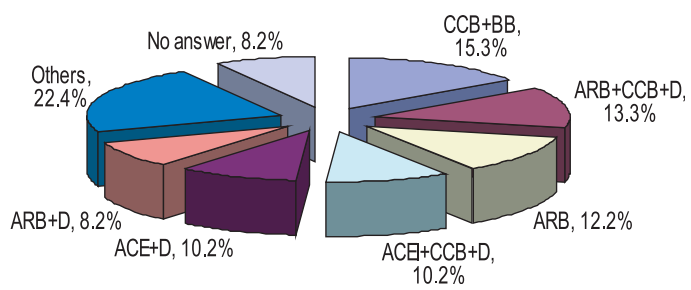

Fig.-6 (B): Antihypertensive utilization in $>55$ years old severe hypertensive patients (Female)

Pittrow D, Kirch W et. al. ${ }^{7}$ studied the pattern of antihypertensive drug usage in Germany in 2001. They analyzed the prescription frequencies of the various antihypertensive drugs in individual patients. However, they did not take the criteria of severity of the hypertension and it was not limited to cardiologists. They have found that ACEI is the mostly used antihypertensive drug class for both males and females aged $>60$ years $(48.4 \%$ in males and $44.3 \%$ females) and BB is the mostly used antihypertensive drug class for both males and females aged $41-60$ years (38\% in males and $41.1 \%$ females). The main difference between this study and our survey is that we are using $\mathrm{BB}$ at a lesser frequency.

Nelson CR, Knapp DA. et. al. ${ }^{8}$ assessed the trends of antihypertensive drugs usage among the ambulatory patients from 1980 to 1995 in United States of America (USA). It was a nation-wide survey conducted by National Center for Health Statistics and Centers for Disease Control and Prevention of USA. US office-based physicians were involved here to collect the data from the hypertensive patients during ambulatory patients' visits. It was a generalized survey and criteria like severity of the hypertension, gender or age difference was not considered. According to this survey, CCB and ACEI/ARB were the most 
commonly used antihypertensive drug classes in 1995. CCB usage had increased from 1.9\% in 1985 to $39.8 \%$ in 1995 while ACEI/ARB usage had increased from $6.4 \%$ to $37.0 \%$, respectively. Our survey differs from this survey on the fact that we have assessed only the current patterns of antihypertensive drug usage while this survey assessed the trend as well.

\section{Conclusion:}

The pattern of using antihypertensive drugs among the cardiologists of Bangladesh in initiating hypertension treatment varies depending on age and gender of the patient and on the severity of hypertension. But, the overall pattern of using antihypertensive drugs did not significantly vary between male and female patients.

\section{References:}

1. Kearney PM, Whelton M, Reynolds K, Muntner P, Whelton PK, He J. Global burden of hypertension: analysis of worldwide data. Lancet 2005; 365(9455): 217 23.

2. Pereira M, Lunet N, Azevedo A, Barros H. Differences in prevalence, awareness, treatment and control of hypertension between developing and developed countries. J Hypertens 2009 May;27(5):963-75.

3. Ezzati M, Lopez AD, Rodgers A, Vander Hoorn S, Murray CJL. Comparative risk assessment collaborative group: selected major risk factors and global and regional burden of disease. Lancet 2002; 360:1347-1360.

4. Zaman MM, Choudhury SR, Ahmed J, Numan SM, Islam MS, Yoshiike N. Non-biochemical risk factors for cardiovascular disease in general clinic-based rural population of Bangladesh. J. Epidemiol 2004 Mar;14(2): 63-8.

5. Volpe M, Tocci G. Managing hypertension in cardiology practice according to risk profile. Int J Clin Pract 2008 Sep;62(9):1403-12. Epub 2008 Jul 24.

6. Mancia G, De Backer G, Dominiczak A, Cifkova R, Fagard R, Germano G, et. Al. 2007 Guidelines for the management of arterial hypertension: The Task Force for the Management of Arterial Hypertension of the European Society of Hypertension (ESH) and of the European Society of Cardiology (ESC). Eur Heart $J$ 2007 Jun;28(12):1462-536.

7. Pittrow D, Kirch W, Bramlage P, Lehnert H, Höfler M, Unger T, Sharma AM, Wittchen HU. Patterns of antihypertensive drug utilization in primary care. Eur J Clin Pharmacol 2004 Apr;60(2):135-42.

8. Nelson CR, Knapp DA. Trends in antihypertensive drug therapy of ambulatory patients by US office-based physicians. Hypertension 2000 Oct;36(4):600-3. 